\title{
ERRATUM
}

doi:10.1038/nature26164

\section{Erratum: The Beaker phenomenon and the genomic transformation of northwest Europe}

Iñigo Olalde, Selina Brace, Morten E. Allentoft, Ian Armit, Kristian Kristiansen, Thomas Booth, Nadin Rohland, Swapan Mallick, Anna SzécsényiNagy, Alissa Mittnik, Eveline Altena, Mark Lipson, Iosif Lazaridis, Thomas K. Harper, Nick Patterson, Nasreen Broomandkhoshbacht, Yoan Diekmann, Zuzana Faltyskova, Daniel Fernandes, Matthew Ferry, Eadaoin Harney, Peter de Knijff, Megan Michel, Jonas Oppenheimer, Kristin Stewardson, Alistair Barclay, Kurt Werner Alt, Corina Liesau, Patricia Ríos, Concepción Blasco, Jorge Vega Miguel, Roberto Menduiña García, Azucena Avilés Fernández, Eszter Bánffy, Maria Bernabò-Brea, David Billoin, Clive Bonsall, Laura Bonsall, Tim Allen, Lindsey Büster, Sophie Carver, Laura Castells Navarro, Oliver E. Craig, Gordon T. Cook, Barry Cunliffe, Anthony Denaire, Kirsten Egging Dinwiddy, Natasha Dodwell, Michal Ernée, Christopher Evans, Milan Kuchařík, Joan Francès Farré, Chris Fowler, Michiel Gazenbeek, Rafael Garrido Pena, María Haber-Uriarte, Elżbieta Haduch, Gill Hey, Nick Jowett, Timothy Knowles, Ken Massy, Saskia Pfrengle, Philippe Lefranc, Olivier Lemercier, Arnaud Lefebvre, César Heras Martínez, Virginia Galera Olmo, Ana Bastida Ramírez, Joaquín Lomba Maurandi, Tona Majó, Jacqueline I. McKinley, Kathleen McSweeney, Balázs Gusztáv Mende, Alessandra Modi, Gabriella Kulcsár, Viktória Kiss, András Czene, Róbert Patay, Anna Endrődi, Kitti Köhler, Tamás Hajdu, Tamás Szeniczey, János Dani, Zsolt Bernert, Maya Hoole, Olivia Cheronet, Denise Keating, Petr Velemínský, Miroslav Dobeš, Francesca Candilio, Fraser Brown, Raúl Flores Fernández, Ana-Mercedes HerreroCorral, Sebastiano Tusa, Emiliano Carnieri, Luigi Lentini, Antonella Valenti, Alessandro Zanini, Clive Waddington, Germán Delibes, Elisa Guerra-Doce, Benjamin Neil, Marcus Brittain, Mike Luke, Richard Mortimer, Jocelyne Desideri, Marie Besse, Günter Brücken, Mirosław Furmanek, Agata Hałuszko, Maksym Mackiewicz, Artur Rapiński, Stephany Leach, Ignacio Soriano, Katina T. Lillios, João Luís Cardoso, Michael Parker Pearson, Piotr Włodarczak, T. Douglas Price, Pilar Prieto, PierreJérôme Rey, Roberto Risch, Manuel A. Rojo Guerra, Aurore Schmitt, Joël Serralongue, Ana Maria Silva, Václav Smrčka, Luc Vergnaud, João Zilhão, David Caramelli, Thomas Higham, Mark G. Thomas, Douglas J. Kennett, Harry Fokkens, Volker Heyd, Alison Sheridan, KarlGöran Sjögren, Philipp W. Stockhammer, Johannes Krause, Ron Pinhasi, Wolfgang Haak, Ian Barnes, Carles Lalueza-Fox \& David Reich

Nature 555, 190-196 (2018); doi:10.1038/nature25738

In this Article, the surname of author Alessandra Modi was incorrectly listed as 'Mod'. This has been corrected online. 